\title{
ANALYSIS OF EFFECTIVNESS OF MAGNETICALLY LABELED BIOSORBENT OBTAINED THROUGH THE MECHANICAL AND MAGNETOHYDRODYNAMIC STIRRING
}

\section{Svitlana Gorobets}

Department of bioinformatics, faculty of Biotechnology and Bioengineering National Technical University of Ukraine "Igor Sikorsky Kyiv Polytechnic Institute" 37 Peremohy ave., Kyiv, Ukraine, 03056

$$
\text { pitbm@ukr.net }
$$

\section{Oksana Gorobets}

Department of bioinformatics, faculty of Biotechnology and Bioengineering National Technical University of Ukraine "Igor Sikorsky Kyiv Polytechnic Institute" 37 Peremohy ave., Kyiv, Ukraine, 03056

Yulia Chyzh

Department of bioinformatics, faculty of Biotechnology and Bioengineering National Technical University of Ukraine "Igor Sikorsky Kyiv Polytechnic Institute" 37 Peremohy ave., Kyiv, Ukraine, 03056

chyzhym@gmail.com

\section{Oleksiy Kovalev}

Department of bioinformatics, faculty of Biotechnology and Bioengineering National Technical University of Ukraine "Igor Sikorsky Kyiv Polytechnic Institute" 37 Peremohy ave., Kyiv, Ukraine, 03056 alexej.covalew@yandex.ua

\section{Valeriya Perizhok}

Department of bioinformatics, faculty of Biotechnology and Bioengineering National Technical University of Ukraine "Igor Sikorsky Kyiv Polytechnic Institute" 37 Peremohy ave., Kyiv, Ukraine, 03056 valeriiazheleva@gmail.com

\section{Volodymyr Golub}

Department of magnetic dynamics of condensed matter Institute of Magnetism

36-b Vernadsky blvd., Kyiv, Ukraine, 03142

golub@imag.kiev.ua

Abstract

The efficiency of $\mathrm{Cu}^{2+}$ ion extraction of magnetically labeled biosorbent based on yeast Sacharomyces cerevisiae obtained by magnetohydrodynamic stirring in combined magnetic and electric fields has been determined. The effect of different concentration of magnetic nanoparticles and yeast cells on the magnetic susceptibility was determined. Optimal magnetite content is $1 \%$, as such biosorbent has a maximum magnetic susceptibility and the best stability. The higher sorption capacity is observed for magnetically labeled biosorbent obtained through the magnetohydrodynamic stirring in the combined magnetic and electric field with concentration of magnetite $1 \%$.

The process of active and passive biosorption shows that part of the magnetite nanoparticles has been attached to the surface of biosorbent. This indicates that both passive and active sorption takes place in the process of interaction of yeast cells and magnetite nanoparticles.

Keywords: magnetically labeled biosorbent, biosorption, magnetic nanoparticles, magnetohydrodynamic stirring. 


\section{Introduction}

Combined treating of waste waters using mechanical, physical and biological methods is the most widespread among the existing technologies of water purification from ferrous and non-ferrous metal impurities, mechanical engineering and electroplating industry [1].

Nowadays the existing biological methods of the waste water purification based on capability of microorganisms to accumulate and absorb ions of heavy metals are spreading very fast [2]. Magnetically labeled biosorption is the most promising method of purification of liquid wastes from the heavy metals among the others [3]. First of all, this method is low-priced because derivative products of biotechnological manufacture can be used as biosorbent (BS). Besides, magnetically labeled BS (MLBSs) can be easily separated from the aqueous solution using the high-gradient magnetic separation (HGMS) in a high-speed mode [4].

Culture of bakery yeasts Saccharomyces cerevisiae has been provided with magnetic properties using magnetic nanoparticles and used as MLBSs in this paper.

\section{Analysis of published data and problem definition}

Significant distribution methods of extracting heavy metal ions obtained biological methods based on the properties of microorganisms to accumulate or absorb heavy metal ions [5]. Among the promising biosorbents used to remove heavy metals, the yeast Saccharomyces cerevisiae is given the increased attention [6].

Last decade the interest grew to the phenomena occurring in electrolyte solutions under the influence of a magnetic field. The significant amount of reliable experimental data was accumulated in this area of research that has been successfully applied in practice. One of the most studied effects in this area is the effect of mass transfer in crossed electric and magnetic fields [7, 8]. It is well known [9] that mixing of electrolytes usually results in acceleration or change in the nature of chemical and electrochemical processes that occur in them.

So much attention is paid to the method of production of the MLBSs [3]. It is known the method of magnetohydrodynamic stirring observed in the external magnetic field after the introduction of a solution of ferromagnetic elements. The presence of a magnetic field leads to the displacement of water solutions of the diffusion layer around the ferromagnetic elements. Stirring is carried out by ferromagnetic elements, which simultaneously carry out mixing the electrolyte in the vicinity of its surface, and has metal binding sites on the surface and the deposition centers yeast adsorbed on the surface of metal ions from aqueous solutions [10].

But the metod of the magnetohydrodynamic stirring is non-technical, as require frequent changes the ferromagnetic tips in a mixing device for providing magnetic properties for biosorbent.

The purpose of the study was to determine the effectiveness of MLBSs, obtained through the mechanical and magnetohydrodynamic stirring in combined magnetic and electric field with different concentrations of magnetic nanoparticles and yeast cells. The active and passive biosorptions were carried out.

\section{Materials and methods}

\section{1. Production of the MLBSs based on bakery yeasts Saccharomyces cerevisiae}

Bakery yeast manufactured by CJSC "Enzym" was used for the experiments in this research. Wet sorbent was obtained through the stirring of the S. cerevisiae biomass with concentration $8 \times 10^{9}$ cells/litre and with the weight fraction of magnetic nanoparticles to yeast biomass 100:1. $(\mathrm{pH}=2.5)$. Proportions were determined in earlier studies [11].

\section{1. 1. Mechanical stirring method}

$4.00 \mathrm{~g}$ of S. cerevisiae biomass, $99,33 \mathrm{ml}$ of distilled water and $0,67 \mathrm{ml}$ of magnetic liquid were mixed. All the above mentioned components were stirred with mechanical mixer for 10 minutes under the $180 \mathrm{rpm}$ speed. Conditions were determined in earlier studies [11]. 


\section{1. 2. Magnetohydrodynamic stirring method in the combined magnetic and elec-} tric field

$4.00 \mathrm{~g}$ of S. cerevisiae biomass, 99, $33 \mathrm{ml}$ of distilled water and $0,67 \mathrm{ml}$ of magnetic liquid were stirred by means of application of the combined external magnetic and electric fields [12]. The voltage $0.5 \mathrm{~V}$ was applied between two cylindrical steel electrodes and the magnetic field strength was equal to $240 \mathrm{kA} / \mathrm{m}$, time of stirring -6 minutes.

After that both MLBSs were fractionated [4]. Steel nickel plated net (size of cells 0,5 mm) was used as ferromagnetic filter in order to separate magnetically labeled and non-magnetic phases. Conditions were determined in earlier studies [12].

\section{2. Magnetic susceptibility measurement method}

Magnetic susceptibility has been measured on experimental installation for magnetic susceptibility measurement using the radiotechnical resonance [13].

Installation consists of the oscillograph, computable electronic frequency measurer, coil quality (coil Q-factor) measurer and LC-circuit. LC-circuit consists of a capacitor and a solenoid. Cylindrical container for the sample was disposed inside the solenoid. Visual observation of the signal was carried out using the oscillograph. The magnitude of resonance frequency of the circuit was carried out using the frequency measurer. The measurement of magnetic susceptibility was carried out 12-20 Hz frequency (relative error less than 0,2\%) [13].

\section{3. Biosorption method of determination of the effectiveness of $\mathrm{Cu}^{2+}$ ions uptake using MLBSs}

Biosorption was carried out by mechanical stirring over 40 minutes, $\mathrm{pH}=5,5$, concentration of $\mathrm{BS}$ in solution $-4 \mathrm{~g} / 1$, concentration of $\mathrm{Cu}^{2+}$ ions $-50 \mathrm{~g} / \mathrm{l}$. Samples were taken at 2, 5, 10, 20, $40 \mathrm{~min}$ and filtrated in order to extract MLBSs. Conditions were determined in earlier studies [11].

\section{4. Sorption capacity of MLBSs measurement method}

Determination of $\mathrm{Cu}^{2+}$ ion concentration was carried out with the help of ULAB 102 spectrophotometer (China). $\mathrm{Cu}^{2+}$ ion solution is colorless. Determination of $\mathrm{Cu}^{2+}$ ions method is based on reaction of $\mathrm{Cu}^{2+}$ ions with aqueous solution of ammonia: $\mathrm{Cu}^{2+}+4 \mathrm{NH}_{3}=\left[\mathrm{Cu}\left(\mathrm{NH}_{3}\right)_{4}\right]^{2+} \cdot\left[\mathrm{Cu}\left(\mathrm{NH}_{3}\right)_{4}\right]^{2+}$ complex has blue color. Measurement of optical density was carried out at wave length $590 \mathrm{~nm}$, according to standard techniques [14].

\section{5. Determination of MLBSs clusterization degree}

Determination of MLBSs clusterization degree was carried out through the mechanical and magnetohydrodynamic stirring in combined magnetic and electric fields with different weight fractions of magnetic nanoparticles and yeast cells using microscope "MICKMED-5" (Russia) with 1000x magnification. nance

\section{6. Determination of magnetic susceptibility of MLBSs using ferromagnetic reso-}

Ferromagnetic resonance (FMR) is one kind of magnetic resonances. It is a multipurpose method of probing the magnetic materials. FMR gives structural and dynamic information about physical processes occurring in material.

Basic characteristics of FMR are the resonance frequency, resonance field, shape and width of signal determined by collective, multi-electron ferromagnetic nature.

Determination was carried out on the ELEXIS E500 EPR spectrophotometer (Bruker BioSpin GmbH, Germany, X-range). The source of electromagnetic emission and detector are placed in microwave bridge. The sample is placed in resonator between two magnets. Resonator is a metallic vessel which enhances the sample signals. Spectrometer consists of a console which fulfills signal processing and a computer used for monitoring all the spectrometer blocks of spectrum registration and data analyzing. 


\section{Results}

4. 1. Determination of magnetic susceptibility of MLBSs obtained through the mechanical and magnetohydrodynamic stirring in combined magnetic and electric field

In the research the analysis of the samples of MLBSS obtained through the mechanical and magnetohydrodynamic stirring in the combined magnetic and electric field with different correlation of magnetic nanoparticles and yeast cells was carried out. (1\%,2\%, $4 \%, 6 \%, 8 \%, 10 \%)$. As a result the values of magnetic susceptibility were measured for both MLBSs obtained through the mechanical and magnetohydrodynamic stirring in the combined magnetic and electric field Fig. 1.

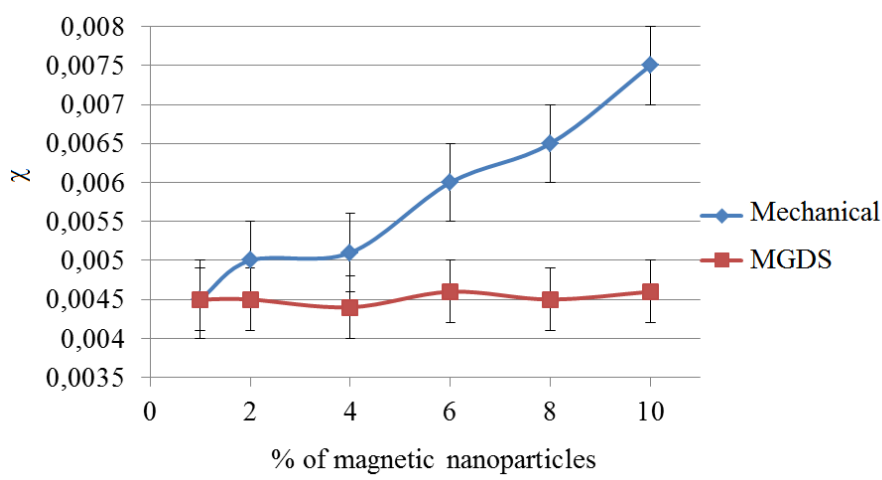

Fig. 1. Magnetic susceptibility $(\chi)$ of MLBSs obtained through the mechanical and magnetohydrodynamic stirring in the combined magnetic and electric field with different weight fractions of magnetic nanoparticles and yeast cells $(1 \%, 2 \%, 4 \%, 6 \%, 8 \%, 10 \%)$

4. 2. Investigation of sorption capacity of MLBSs obtained through the mechanical and magnetohydrodynamic stirring in the combined magnetic and electric field

MLBSs obtained through the magnetohydrodynamic stirring in the combined magnetic and electric field have fewer predispositions to form clusters than the MLBSs obtained through the mechanical stirring Fig. 2. So, such MLBSs have larger surface area with free binding sites for ions of heavy metals and therefore high sorption capacity.

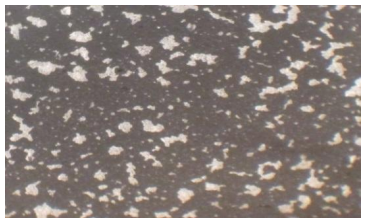

$a$

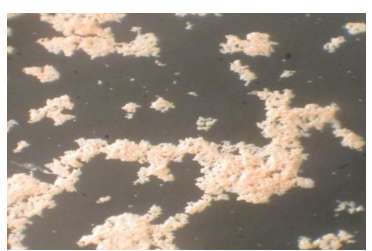

$e$

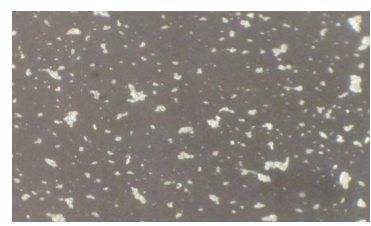

$b$

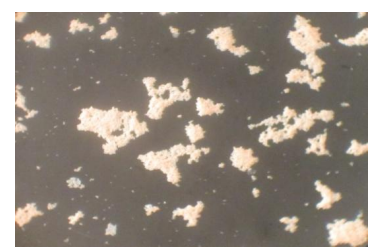

$f$

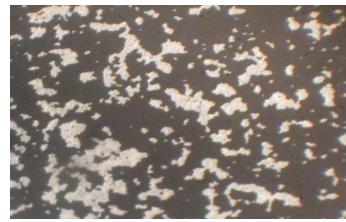

$c$

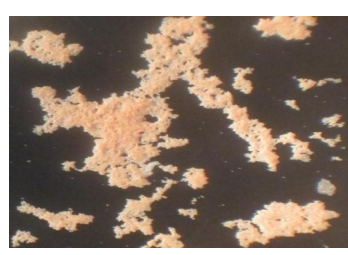

$j$

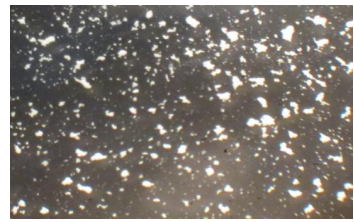

$d$



$h$

Fig. 2. Optical image of MLBS obtained through the mechanical and magnetohydrodynamic stirring in the combined magnetic and electric field with different weight fractions of magnetic nanoparticles and yeast cells (1\%, $3 \%, 7 \%, 10 \%)$ : $a$-mechanical stirring (1\% magnetite); $b$-magnetohydrodynamic stirring in the combined magnetic and electric field (1\% magnetite); $c$ - mechanical stirring (3\% magnetite); $d$-magnetohydrodynamic stirring in the combined magnetic and electric field (3\% magnetite); $e$-mechanical stirring (7\% magnetite); $f$-magnetohydrodynamic stirring in the combined magnetic and electric field (7\% magnetite); $j$ - mechanical stirring (10\% magnetite); $h$ - magnetohydrodynamic stirring in the combined magnetic and electric field (10\% magnetite) 
It is obvious from Fig. 3 that the higher sorption capacity is observed for MLBSs obtained through the magnetohydrodynamic stirring in the combined magnetic and electric field with concentration of magnetite $1 \%$. The lower sorption capacity is observed for MLBSs obtained through the mechanical stirring with concentration of magnetite $10 \%$.

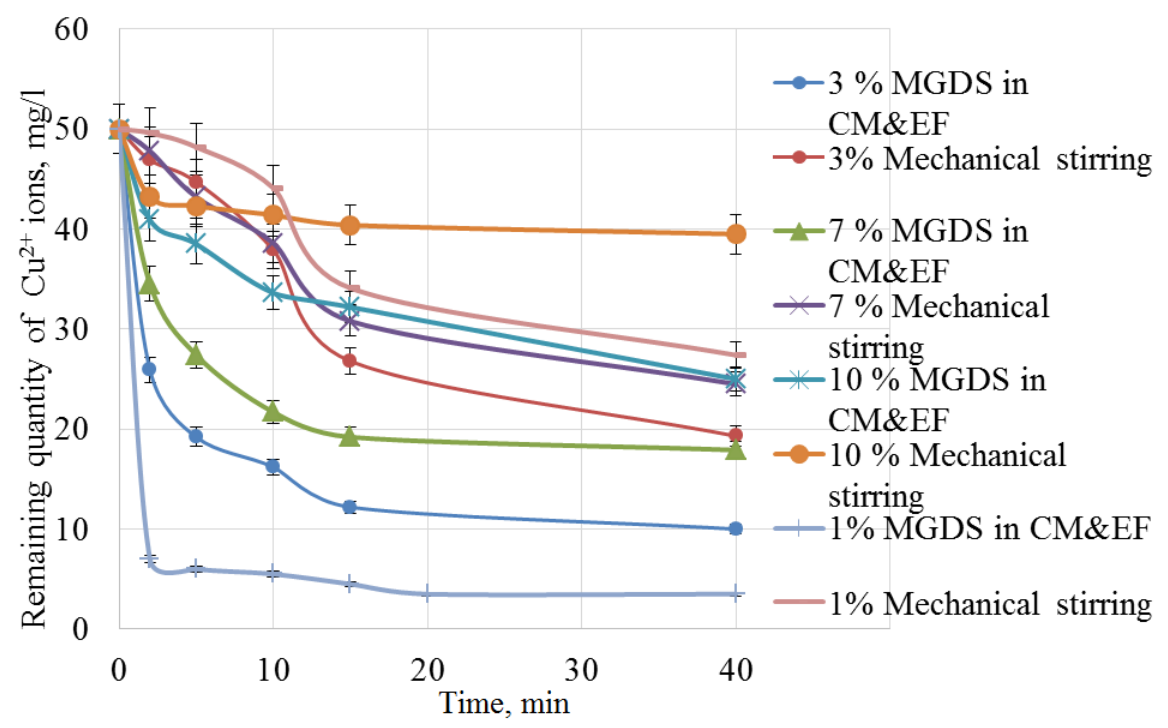

Fig. 3. Sorption capacity of MLBSs, obtained through the mechanical and magnetohydrodynamic stirring in the combined magnetic and electric field (MGDS in CM\&FF) with different weight fractions of magnetic nanoparticles and yeast cells $(1 \%, 3 \%, 7 \%, 10 \%)$

4. 3. Active and passive sorption determination of BS obtained by magnetohydrodynamic stirring in the combined magnetic and electric field

Magnetic susceptibility of magnetically labeled BS obtained by magnetohydrodynamic stirring in the combined magnetic and electric field has decreased after the abstersion with acid solution with $\mathrm{pH}=2$ [15]. This shows that part of the magnetite nanoparticles has been attached to the surface of BS obtained through the mechanical stirring Fig. 4, and obtained through the magnetohydrodynamic stirring in the combined magnetic and electric field Fig. 5. These results indicate that both passive and active sorption takes place in the process of interaction of yeast cells and magnetite nanoparticles.

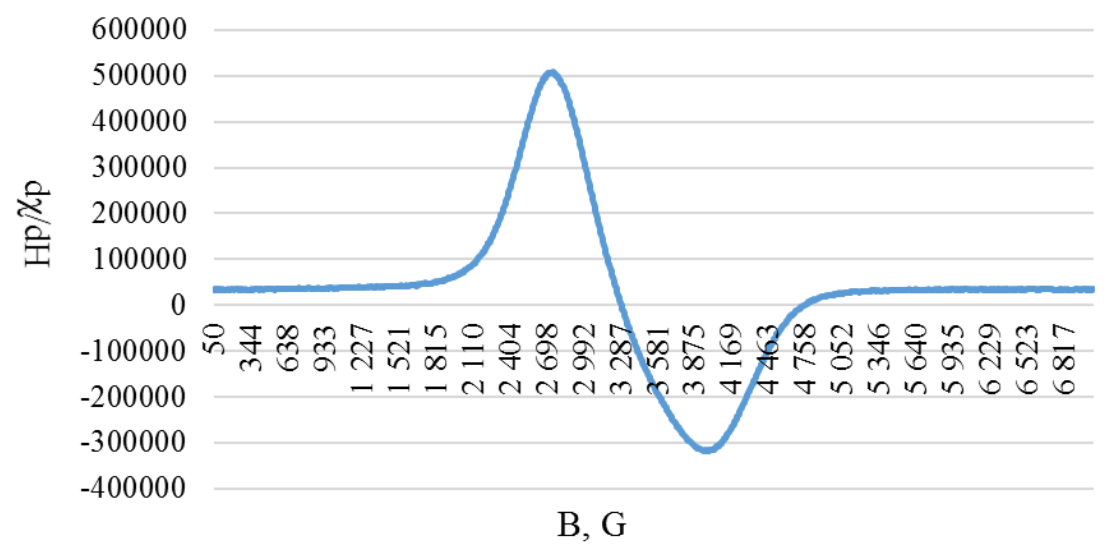

Fig. 4. $1 \%$ fractionated BS obtained through the mechanical stirring. Hp/ $\chi \mathrm{p}-$ the first derivative of energy absorption of electromagnetic radiation by the magnetic field.

$\mathrm{B}-$ magnetic field, which is measured in Gauss 


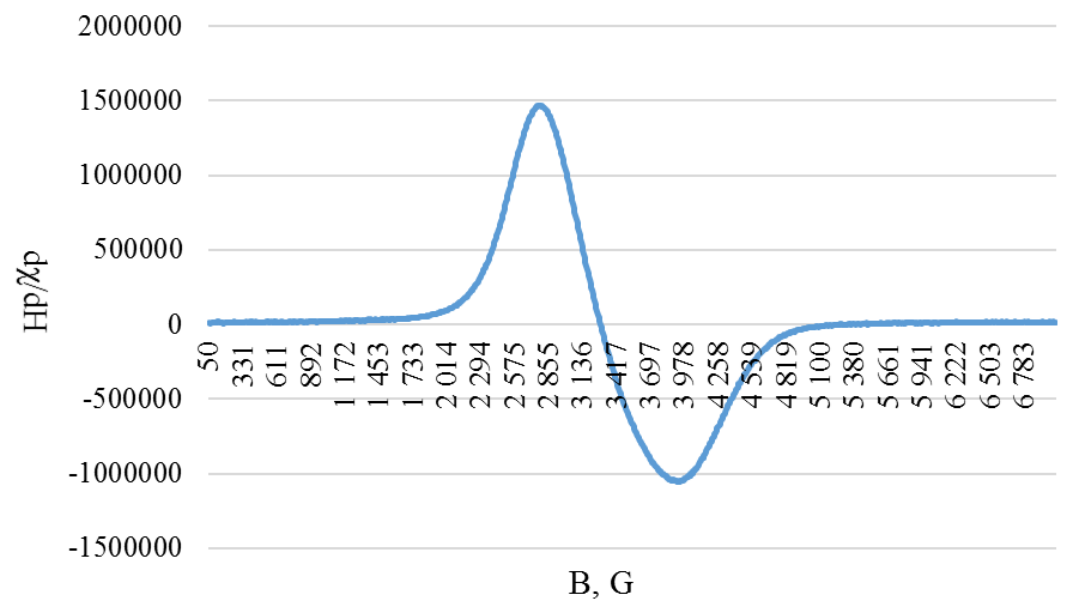

Fig. 5. $1 \%$ fractionated BS obtained through the magnetohydrodynamic stirring in the combined magnetic and electric field. $\mathrm{Hp} / \chi \mathrm{p}$ - the first derivative of energy absorption of electromagnetic radiation by the magnetic field. $\mathrm{B}$ - magnetic field, which is measured in Gauss

Magnetic susceptibility of magnetically labeled BS obtained by magnetohydrodynamic stirring in the combined magnetic and electric field has decreased after the abstersion with acid solution with $\mathrm{pH}=2$. This shows that part of the magnetite nanoparticles has been attached to the surface of BS.

\section{Discussion of research results}

Magnetic susceptibility of BS obtained through the magnetohydrodynamic stirring in the combined magnetic and electric field remained constant for the samples with different concentrations of magnetic nanoparticles.

Considering this the conclusion was made that MLBS obtained through the magnetohydrodynamic stirring in the combined magnetic and electric field has more stable magnetic characteristics. That is why it seems obvious that using BS with higher concentration of magnetic nanoparticles than $1 \%(100: 1)$ is useless.

Magnetic susceptibility of BS obtained through the mechanical stirring was higher than of BS obtained through the magnetohydrodynamic stirring in the combined magnetic and electric field and was increasing with increase of concentration of magnetic nanoparticles. So BS obtained through the mechanical stirring has less stable magnetic characteristics.

MLBSs, obtained through the mechanical stirring has higher clusterization degree, smaller surface area with free binding sites for ions of heavy metals and therefore low sorption capacity.

The results correlate with the conclusion that the rise of the clusterization degree in analyzed MLBSs causes decrease of the sorption capacity for both BS obtained through the mechanical and magnetohydrodynamic stirring in the combined magnetic and electric field. But it has been shown that the degree of clusterization for the MLBSs obtained through the mechanical stirring is much higher than MLBSs obtained through the magnetohydrodynamic stirring in the combined magnetic and electric field.

After 10 minutes of the sorption process using BS obtained in the combined magnetic and electric field the uptake of copper ions from solution is $60 \%$ higher than using BS obtained by mechanical stirring. Obviously (Fig. 3), sorption capacity reaches $90 \%$ of the initial concentration of $\mathrm{Cu}^{+2}$ ions in solution after 20 minutes.

\section{Conclusions}

The effect of different concentration of magnetic nanoparticles and yeast cells on the magnetic susceptibility was determined. Optimal magnetite content is $1 \%$, as such BS has a maximum magnetic susceptibility and the best stability. The higher concentration of magnetic nanoparticles caused the destruction of the cell wall, therefore loss of magnetic susceptibility and instability of obtained BS because of desorption of magnetite nanoparticles into the solution. 
The sorption capacity of MLBS obtained through mechanical stirring and magnetohydrodynamic stirring in combined electric and magnetic fields was determined. BS obtained through magnetohydrodynamic stirring in combined electric and magnetic fields has higher effectiveness than obtained by mechanical stirring. That confirms the feasibility of magnetohydrodynamic stirring in combined electric and magnetic fields method.

The higher sorption capacity is observed for MLBSs obtained through the magnetohydrodynamic stirring in the combined magnetic and electric field with concentration of magnetite $1 \%$. The lower sorption capacity is observed for MLBSs obtained through the mechanical stirring with concentration of magnetite $10 \%$.

The process of active and passive biosorption was carried out. Magnetic susceptibility of magnetically labeled BS obtained by magnetohydrodynamic stirring in the combined magnetic and electric field has decreased after the abstersion with acid solution with $\mathrm{pH}=2$. This shows that part of the magnetite nanoparticles has been attached to the surface of BS. This indicates that both passive and active sorption takes place in the process of interaction of yeast cells and magnetite nanoparticles.

Magnetically labeled method is used not only for purification of waste water but also for drug delivery to the target organ. Therefore, today it is the actual problem the even magnetic labeling of microorganisms, liposomes etc.

\section{References}

[1] Barakat, M. A. (2011). New trends in removing heavy metals from industrial wastewater. Arabian Journal of Chemistry, 4 (4), 361-377. doi: 10.1016/j.arabjc.2010.07.019

[2] Javanbakht, V., Alavi, S. A., Zilouei, H. (2014). Mechanisms of heavy metal removal using microorganisms as biosorbent. Water Science \& Technology, 69 (9), 1775. doi:10.2166/wst.2013.718

[3] Uzun, L., Sağlam, N., Safarikova, M., Safarik, I., Denizli, A. (2011). Copper Biosorption on Magnetically Modified Yeast Cells Under Magnetic Field. Separation Science and Technology, 46 (6), 1045-1051. doi: 10.1080/01496395.2010.541400

[4] Gorobets, S. V., Mikhailenko, N. A. (2014). High-gradient ferromagnetic tips for sewage treatment, obtained by magnitoelektroliz. Chemistry and technology of water, 4, 283-296.

[5] Michalak, I. K. Chojnack, A. (2013). Witek-Krowiak State of the Art for the Biosorption Process a Review. Appl Biochem Biotechnol., 170, 1389-1416.

[6] Aksu, Z. (2005). Application of biosorption for the removal of organic pollutants: a review. Process Biochemistry, 40 (3-4), 997-1026. doi: 10.1016/j.procbio.2004.04.008

[7] Tacken, R. A., Janssen, L. J. J. (1995). Applications of magnetoelectrolysis. Journal of Applied Electrochemistry, 25 (1). doi: 10.1007/bf00251257

[8] Waskaas, M., Kharkats, Y. I. (1999). Magnetoconvection Phenomena: A Mechanism for Influence of Magnetic Fields on Electrochemical Processes. The Journal of Physical Chemistry B, 103 (23), 4876-4883. doi: 10.1021/jp984730t

[9] Gorobets, S. V., Gorobets, O. Yu., Bandurka, N. P. (2001). Stationary flows of liquid in the vicinity of the small ferromagnetic particles in constant homogeneous magnetic field. The Physics of Metals and Metallography, 92, 197-198.

[10] Gorobets, S. V., Gorobets, O. Yu., Reshetnyak, S. A. (2003). Electrolyte vortex flows induced by a steady-state magnetic field in the vicinity of a steel wire used as an accelerator of the chemical reaction rate. Magnetohydrodynamics, 39, 211-214.

[11] Gorobets, S. V., Chyzh, Y. M., Kovalev, O. V., Shpetnyi, I. O. (2015). The effectiveness of magnetically biosorbent from yeast Saccharamyces cerevisiae for waste water treatment. Naukovi visti NTUU "KPI", 3.

[12] Gorobets, S. V., Gorobets, O. Yu., Chyzh, Yu. M., Kovalyov, O. V. Method of getting of magnetically biosorbent. Patent for utility model 101016.

[13] Barbeta, V. B., Jardim, R. F., Kiyohara, P. K. (2010). Magnetic properties of $\mathrm{Fe}_{3} \mathrm{O}_{4}$ nanoparticles coated with oleic and dodecanoic acids. Nanoscale Physics, 1, 1-8

[14] Krasnov, T., Voronyuk, I. (2011). Analytical chemistry. A teaching manual for higher education, 20-24.

[15] Neuhoff, S., Ungell, A.-L., Zamora, I., Artursson, P. (2005). pH-Dependent passive and active transport of acidic drugs across Caco-2 cell monolayers. European Journal of Pharmaceutical Sciences, 25 (2-3), 211-220. doi: 10.1016/j.ejps.2005.02.009 